Research Paper

\title{
Distinct Changes in the Expression TAZ are Associated with Normal Cervix and Human Cervical Cancer
}

\author{
Yaofang Liu' ${ }^{1}$, Meiping Ren ${ }^{2}$, Xiaoyong Tan ${ }^{2}$, Lina $\mathrm{Hu}^{1}$ \\ 1. Department of Gynecology and Obstetrics, The Second Affiliated Hospital of Chongqing Medical University, Chongqing, 400010, P.R. China \\ 2. Drug Discovery Research Center, Southwest Medical University, Luzhou, Sichuan, 646000, P.R. China \\ $\bowtie$ Corresponding author: Lina Hu, cyfeyhulina@163.com \\ (c) Ivyspring International Publisher. This is an open access article distributed under the terms of the Creative Commons Attribution (CC BY-NC) license \\ (https://creativecommons.org/licenses/by-nc/4.0/). See http://ivyspring.com/terms for full terms and conditions.
}

Received: 2018.04.11; Accepted: 2018.08.28; Published: 2018.10.20

\begin{abstract}
The transcriptional coactivator with the PDZ-binding motif (TAZ) has been associated with different types of cancer. In this study, we examined the TAZ protein expression and cellular localization in 194 cases of human cervical squamous cell carcinoma (SCC). We observed that a normal cervix is characterized by higher expression levels of both nuclear and cytosolic TAZ compared to cervical SCC. Lower membranous and cytosolic TAZ expression levels are associated with lymph node involvement. We observed that TAZ expression levels are associated with $\beta 1$ integrin and Src in SCC and cell lines derived from human cervical cancers. Of note, knock down of TAZ increased the expression of $\beta 1$ integrin and Src in both normal and human cervical cancer cells. Our data indicate that the expression and cellular localization of TAZ are inversely associated with the development and progression of cervical SCC, and TAZ-mediated transcription may be involved in the activation of the integrin-Src signalling pathway.
\end{abstract}

Key words: cervix, SCC, TAZ (Hippo) signaling, integrin

\section{Introduction}

Cervical cancer (CC) is the second most common cancer and the fourth leading cause of cancerassociated mortality in women worldwide [1]. Squamous cell carcinoma (SCC) of the cervix is one of the most common pathological types of CC. The development of invasive SCC of the cervix involves the progression of premalignant lesions and is associated with persistent infection and oncogenes $[2,3]$. Biomarkers that can predict the progression of $\mathrm{CC}$ is an area of great clinical interest. The molecular mechanisms underlying cervical cancer initiation and progression are still largely unknown.

The Hippo signalling pathway is an evolutionarily conserved pathway that controls organ size and functions as a critical regulator of cell proliferation, self-renewal, differentiation and survival [4]. Moreover, Hippo signalling exerts both tumoursuppressive and oncogenic activities in several human malignancies [5,6]. TAZ (transcriptional coactivator with PDZ-binding motif) and YAP
(Yes-associated protein) are the major downstream effectors of the hippo pathway and serve as transcriptional co-activators. The activation of TAZ has been associated with a variety of tumourpromoting functions. Phosphorylated TAZ undergoes degradation and/or is sequestered in the cytoplasm. Conversely, elevated TAZ expression and nuclear localization have been observed in a broad range of different human cancers, including lung, breast, gastric, and liver cancer [7-10], and the change often correlates with poor patient prognosis.

Nevertheless, little is known on the role of the TAZ in the Hippo pathway of cervical cancer. The previous study demonstrated that increased levels of TAZ were detected in cervical cancer and its associated microenvironments [11]. In cervical cancer, TAZ is a potentially relevant Hippo effector because TAZ, and not YAP protein expression in cancer cells, was associated with a reduced pathological complete response (pCR) [11]. However, the precise mechanism 
for the expression and function of TAZ in cervical cancer cell remains undefined.

Integrins play an important role in tumour growth and metastasis [12]. Previous studies have shown the effects of $\beta 1$ integrin expression on cervical SCC tissues, including its association with clinical stages and degrees of malignancy [13]. However, at present, the association between integrin activity regulation and cancer is not fully elucidated. The studies have linked integrin-Src signalling and the Hippo pathway YAP/TAZ signalling axis to cancer progression and metastasis; in addition, studies have shown that this process is regulated by activating a $\beta 1$ integrin/RhoGTPase signalling cascade and triggering the nuclear localization of the transcriptional coactivators YAP and TAZ $[14,15]$. Moreover, a recent study demonstrated that $\beta 1$ integrin and YAP/TAZ proteins are linked to prognosis and therefore could be therapeutic targets [16]. To better understand the role of TAZ in the development and progression of cervical cancer, we analysed 194 human SCC samples by establishing a linking between the expression and localization of TAZ to integrin-Src signalling. We also explored the role of Src in the translocation of nuclear TAZ in cultured human cervical cancer cells. Our data indicated that the expression of TAZ is inversely correlated with human cervical cancer progression. TAZ is associated with integrin-Src signalling in SCC.

\section{Materials and methods}

\section{Patients and tissue specimen}

The patient study was conducted in accordance with the Declaration of Helsinki, and the study protocol was approved by the Ethics Committee of the Affiliated Hospital of Southwest Medical University, China. All patients provided written informed consent. The 194 research subjects were Han Chinese individuals who participated in cervical cancer screening between 2014 and 2017 at the Affiliated Hospital of Southwest Medical University. A total of 194 histologically confirmed cervical cancer patients were selected, including 141 cases of SCC and 53 cases of SCC with lymph node metastases. Eleven cases of normal cervix samples were selected from a cohort of patients who were screened for cervical cancer through conventional methods, including Pap smears, naked eye visual inspections with acetic acid (VIA) and Lugol's iodine (VILI), HPV testing for high-risk HPV, and biopsies.

\section{Cell lines}

ME180, and Ect1/E6E7 cells were purchased from ATCC (Manassas, VA). ME180 cells were derived from an omental metastasis of a recurrent cervical epidermoid carcinoma [17]. Ect1/E6E7 cell line was established from normal epithelial tissue taken from a premenopausal woman undergoing hysterectomy for endometriosis [18]. The cells used in this study were from passages 6-12.

\section{Immunofluorescence and Confocal Microscopy}

TAZ and $\beta 1$ integrin protein expression levels in human cervical tissues were detected using immunofluorescence staining as described previously [19]. Briefly, the tissue blocks were embedded in Tissue-Tek OCT compound and quickly frozen in a dry ice acetone bath. Frozen sections $(5 \mu \mathrm{m})$ were collected on silane-coated glass slides. TAZ and $\beta 1$ integrin were detected with rabbit anti-human TAZ polyclonal antibodies and rabbit anti-human $\beta 1$ integrin polyclonal antibodies, respectively. The secondary antibody was Alexa 546 goat anti-rabbit IgG (Thermo Fisher, Waltham, MA). To analyse the precise localization and amount of TAZ, $\beta 1$ integrin immunostaining was conducted, images of crosssections were captured under identical conditions and imported into the IMAGE-PRO PLUS software. The images were recorded at $\times 20$ or $\times 40$ magnifications, and the threshold image intensity was adjusted for measurement in pixels. Nine to 12 images $(\times 20)$ from three to five tissue sections were analysed.

To further examine the interactions between TAZ, $\beta 1$ integrin, and Src in the normal and SCC. The tissue sections were stained with Rabbit anti-human TAZ (Abcom), Mouse anti-human $\beta 1$-integrin (Abcom), and Sheep anti-human Src (Santa Cruz) followed by secondary antibodies, Goat anti-mouse IgG (H+L)antibody (Alexa Fluor plus 647), Goat anti-rabbit IgG (H+L)antibody (Alexa Fluor plus 555), and Chicken anti-goat IgG (H+L)antibody (Alexa Fluor plus 488). Confocal microscopy (LSM510, Carl Zeiss) was used to acquire images (200×) in a blinded fashion using identical confocal settings for all images. Negative controls (lacking primary antibody but containing the secondary antibody) confirmed the specificity of the staining. For quantification of immunofluorescence intensity, four sections from each tissue were randomly selected, and images were imported into Image (NIH), and positive signal was thresholded to secondary-only controls. The images are quantified from positive signal and presented as mean pixel density per group of samples.

\section{TAZ knockdown}

ME180, and Ect1/E6E7 cells were transfected using Lipofectamine 2000 transfection reagent (Life Technologies) according to manufacturer's guidelines. Validated or sequence-specific siRNAs were 
purchased from Genepharma (Shanghai, China) against the following targets: (i) human TAZ (sense, CCUGCCGGAGUCUUUCUUUTT; antisense, AAAG AAAGACUCCGGCAGGTT); (ii) the control siRNA (sense, UUC UCC GAA CGU GUC ACG UTT; antisense, ACG UGA CAC GUU CGG AGA ATT).

\section{Immunoblotting}

The tissues were homogenized in RIPA buffer (Sigma) and the total protein concentrations of the homogenates were measured using the BCA reagent. Equal amounts of protein were subjected to SDS-PAGE and transferred to polyvinylidene difluoride membranes by electroblotting. After blocking, the membranes were incubated with antibodies against human TAZ (Abcam), human $\beta 1$ integrin (Abcam), and $\beta$-actin (Cell Signalling Technology).

ME180 cells were grown on 100-mm culture dishes until they reached $80 \%$ confluency and then incubated with su6656, an inhibitor of the Src family of kinases $(1 \mu \mathrm{M})$ (Calbiochem. Calif., USA), or a vehicle control (DMSO) for $1 \mathrm{hr}$. The lysates were prepared in RIPA buffer. The cytosolic and nuclear extracts were obtained using NE-PER nuclear and cytoplasmic extraction reagents (Thermo Fisher, Waltham, MA). Equal amounts of protein were subjected to SDS-PAGE and transferred to polyvinylidene difluoride membranes by electroblotting. After blocking, the membranes were incubated with primary TAZ antibodies (Abcam). The secondary antibody was an anti-rabbit IgG conjugated to horseradish peroxidase (Santa Cruz Biotechnology, Inc., Santa Cruz, CA), diluted 1:2000 and incubated with the membrane for $1 \mathrm{~h}$. The protein bands were detected using enhanced chemiluminescence plus detection system according to the manufacturer's instructions (BioRad).

\section{Statistical analysis}

Data are presented as the mean \pm standard error of the mean (SEM). Statistical comparisons between 2 samples were performed using the Student's t-test. Statistical comparisons of more than 2 groups were performed using one-way analysis of variance with Bonferroni's post hoc test. A P-value of less than 0.05 was considered statistically significant.

\section{Results}

\section{Patient characteristics}

The clinico-pathological characteristics of the 194 patients are summarized in Table 1 . The median age was $46.15 \pm 5.91$ years, and the mean tumour size was $18.58 \pm 16.82 \mathrm{~cm}^{3}$. We determined the TAZ expression levels by immunofluorescence staining in 194 consecutive SCC cases. In all, 84.54\% 164/194) of the SCC samples stained positive for TAZ, most of which was located in the cytosolic of tumour cells. Furthermore, nuclear TAZ expression was observed in $36.08 \%$ (70/194). No significant correlations were observed between TAZ positive staining and standard pathological parameters such as tumour size and tumour grade. In contrast, TAZ expression was positively correlated with lymph node status.

Table 1: clinical data of the 194 patients

\begin{tabular}{lll}
\hline Median age & $\mathbf{4 6 . 1 5} \mathbf{\pm 5 . 9 1}$ years & $\mathbf{( 2 8 - 7 1}$ years) \\
\hline Clinical staging & 103 & $(53.09 \%)$ \\
I & 91 & $(46.91 \%)$ \\
IIA & & \\
Histological stage & 61 & $(31.44 \%)$ \\
G1 & 69 & $(35.57 \%)$ \\
G2 & 64 & $(32.99 \%)$ \\
G3 & $18.58 \pm 16.82 \mathrm{~cm}^{3}$ & \\
Mean tumor size & & \\
lymph node metastasis & 141 & $(72.68 \%)$ \\
$\begin{array}{l}\text { Negative* } \\
\text { Positive** }\end{array}$ & 53 & $(27.32 \%)$ \\
\hline *negative=N0 & \\
** positive= $\geq$ N1mi (according to TNM 7th edition, 2010) &
\end{tabular}

\section{TAZ is expressed in both normal and SCC tissues}

We began to evaluate the expression levels and subcellular localizations of TAZ in both normal cervix and SCC tissues. TAZ was found to be expressed in a subset of cells in the normal and SCC cervical tissues. Immunofluorescence imaging analysis indicated that the expression of TAZ is significantly higher in normal tissues than that in SSC and SCC with metastasis tissues (Fig. 1A-B). The nuclear localization of TAZ was particularly prominent in the basal layer cells of normal cervix, but not in SCC (Fig. 1A). We also found that TAZ expression and localization were related to tumour metastasis. Compared to tumour tissues from patients with lymph node involvements had significantly lower levels of TAZ protein, which was localized mainly in the cytoplasm and membranes of tumour cells (Fig. 1A). Furthermore, nuclear and cytosolic fractions were isolated from normal cervical and SCC tissues, and subjected to western blot analysis to detect TAZ. TAZ was predominantly expressed in both the cytoplasm and the nucleus in normal tissues compared with SCC (Fig. 1C-D). We found a striking restriction of these TAZ targets to the basal layer of the normal cervix, indicating that the expression TAZ is inversely associated with cervical cancer progression.

\section{Expression of $\beta 1$ integrin-Src in SCC}

Recent work in SCC tissues indicates a role for integrin-Src signalling and in promoting the nuclear 
localization of YAP [15]. We examined the expression of $\beta 1$ integrin and Src in normal and SCC tissues by immunoblotting. We found that integrin $\beta 1$ and Src have higher expression levels in SCC compared to normal tissues (Fig. 2A-D). Furthermore, we compared the expression levels and the localization of $\beta 1$ integrin between cervical SCC and SCC with

A
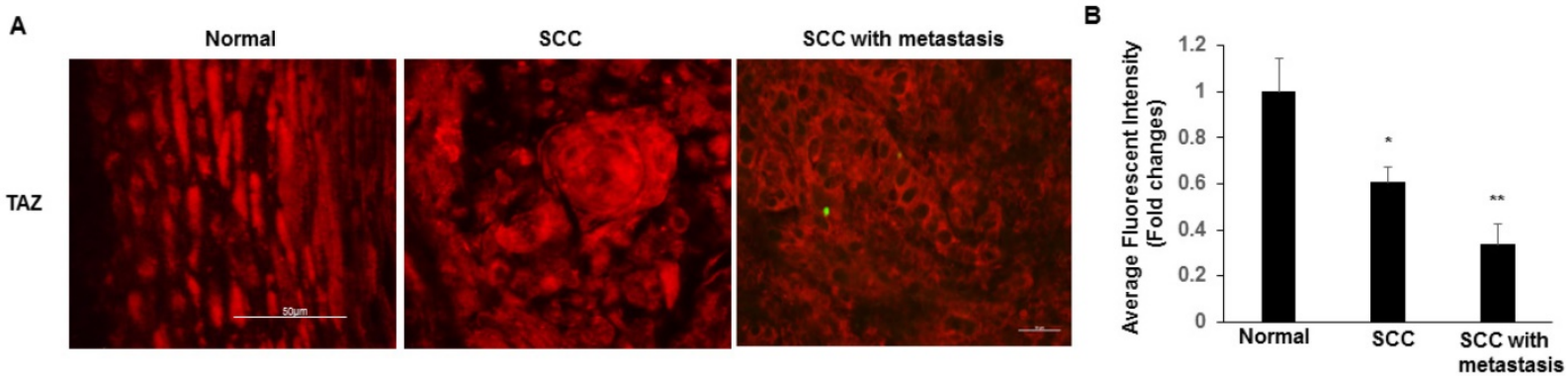

metastasis. Compared to SCC, SCC with metastasis showed more frequent expression of membranous and cytosolic expressions of $\beta 1$ integrin (Fig. 2E-F), which is consist with TAZ expression in SCC with metastasis, suggesting that nuclear TAZ localization might be regulated by integrin-Src in SCCs.
C

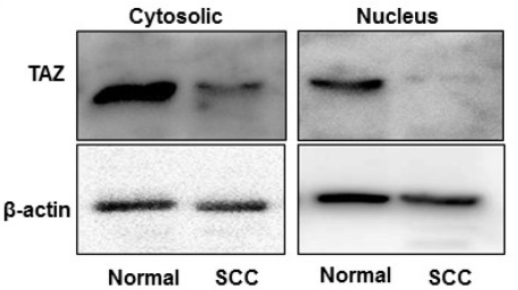

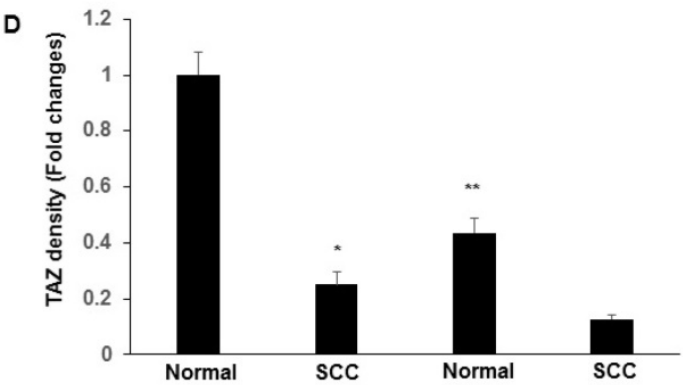

Figure 1.TAZ expression and localization in normal cervical tissues and cervical cancer tissues. A. Representative images show expression of TAZ in normal cervical tissues, SCC, and SCC with metastases. Scale bar: $10 \mathrm{~mm}$. B. Quantification of immunofluorescence fluorescent intensity of normal, SCC, and SCC with metastasis. Data presented as the fold change using Image J (NIH). $* P<0.05$ vs normal; **P<0.05 vs normal and SCC. C. Nuclear and cytosolic fractions were isolated from normal cervical and SCC tissues, and subjected to western blot analysis to detect TAZ. The levels of $\beta$-actin were used as internal protein loading controls. D. The graph represents densitometric analyses of 3 independent experiments. $* P<0.05$ vs cytosolic fraction from normal; $* * P<0.05$ vs cytosolic fraction from SCC.

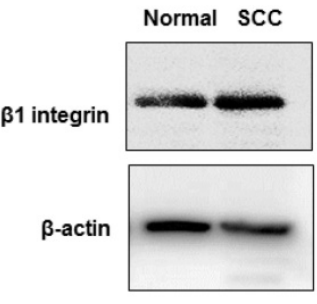

B

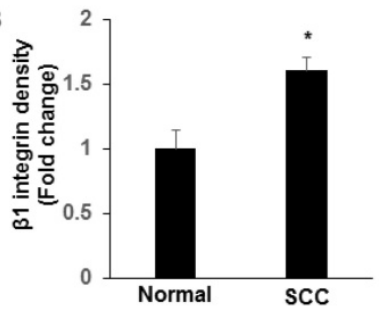

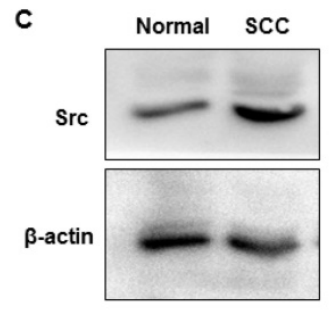

D

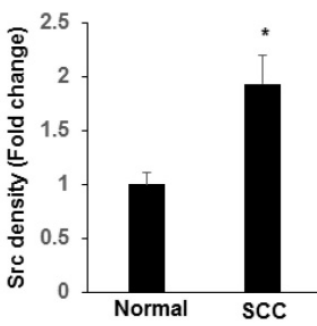

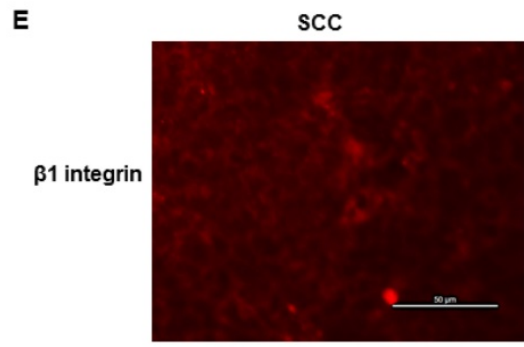

SCC with metastasis
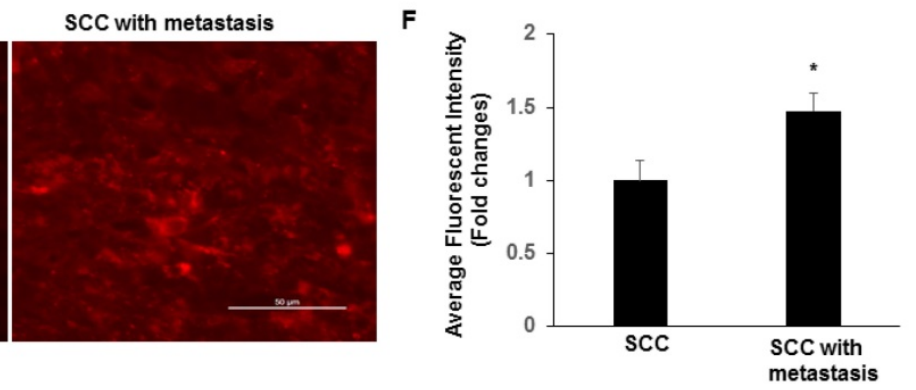

Figure 2. Expression of $\beta 1$ integrin-Src in normal and SCC cervical tissues. Tissues lysates were prepared from normal cervical and SCC tissues, and subjected to western blotting to detect $\beta 1$ integrin (A-B) and Src (C-D). The levels of $\beta$-actin were used as internal protein loading controls. All graphs correspond to the blots above them and represent densitometric analyses of 3 independent experiments. $* P<0.05$ vs normal. E-F. Representative images show the expression of $\beta 1$ integrin in SCC and SCC with metastasis tissues with quantification of fluorescence intensity. $* P<0.05$ vs normal; Scale bar: $50 \mathrm{~mm}$. 


\section{Src signalling is required for TAZ localization}

The expressions of TAZ, Src and $\beta 1$ integrin in ME180 cell line were characterized by immuneblotting. Immunoblotting results shown in Fig. 3A-B indicate that the cervical cancer cell line, ME180, show increased expression of $\beta 1$ integrin and Src compared to Ect1/E6E7 cells. To confirm that the integrin-Src signalling pathway is required for TAZ nuclear localization in cervical cancer cells, we manipulated the Src signalling pathway by treating the ME180 cells with an inhibitor of Src family kinases, su6656. We determined the TAZ levels in cytosolic and nuclear extracts. As shown in Fig. 3C-D, the levels of TAZ in cytosolic and nuclear extracts show a pattern similar to that observed in SCC tissue extracts. The su6656 cells showed decreased TAZ levels in their cytosolic extracts, and increased TAZ levels in their nuclear fractions, indicating that the localization of TAZ is associated with the level of activation of the Src signalling pathway.

We further examined whether TAZ was linked to the downstream signal, $\beta 1$ integrin and Src. Ect1/E6E7 and ME 180 cells were transfected with either control siRNA or TAZ siRNA. As shown in Fig. 3E-F, transfected with TAZ siRNA showed increased the expression of $\beta 1$ integrin and Src in both Ect1/E6E7 and ME 180 cells, suggesting a close regulatory link between TAZ, $\beta 1$ integrin, and Src.

\section{Subcellular localization of TAZ, $\beta 1$ integrin and Src}

Normal cervix tissues expressed substantially more TAZ than SCC tissues, specifically in nuclear fractions (Fig. 4A-B). $\beta 1$ integrin and Src were more highly expressed in SCC than in normal tissues. In contrast, coexpression of TAZ, $\beta 1$ integrin and Src was markedly absent in SCC compared with that in normal tissues, and with a specific increase in SCC in the cytoplasm fractions.

\section{Discussion}

Previous studies reported that TAZ is expressed in different types of cancers [11.20-22]. The purpose of this study was to investigate the expression of TAZ in cervical SCC. Immunofluorescence staining of TAZ demonstrated that higher expression levels of both nuclear and cytosolic TAZ were observed in all normal cervix tissues. Nuclear TAZ expression was observed in $36.08 \%$ of all patients with SCC. Low levels of membranous and cytosolic TAZ expression and no nuclear expression were associated with SCC that had lymph node involvement. It should be noted that the cellular localization of TAZ reflects ubiquitin-mediated proteolysis, whereas nuclear translocation is usually interpreted as an activation of the TAZ-mediated oncogenic transcriptional program [4].
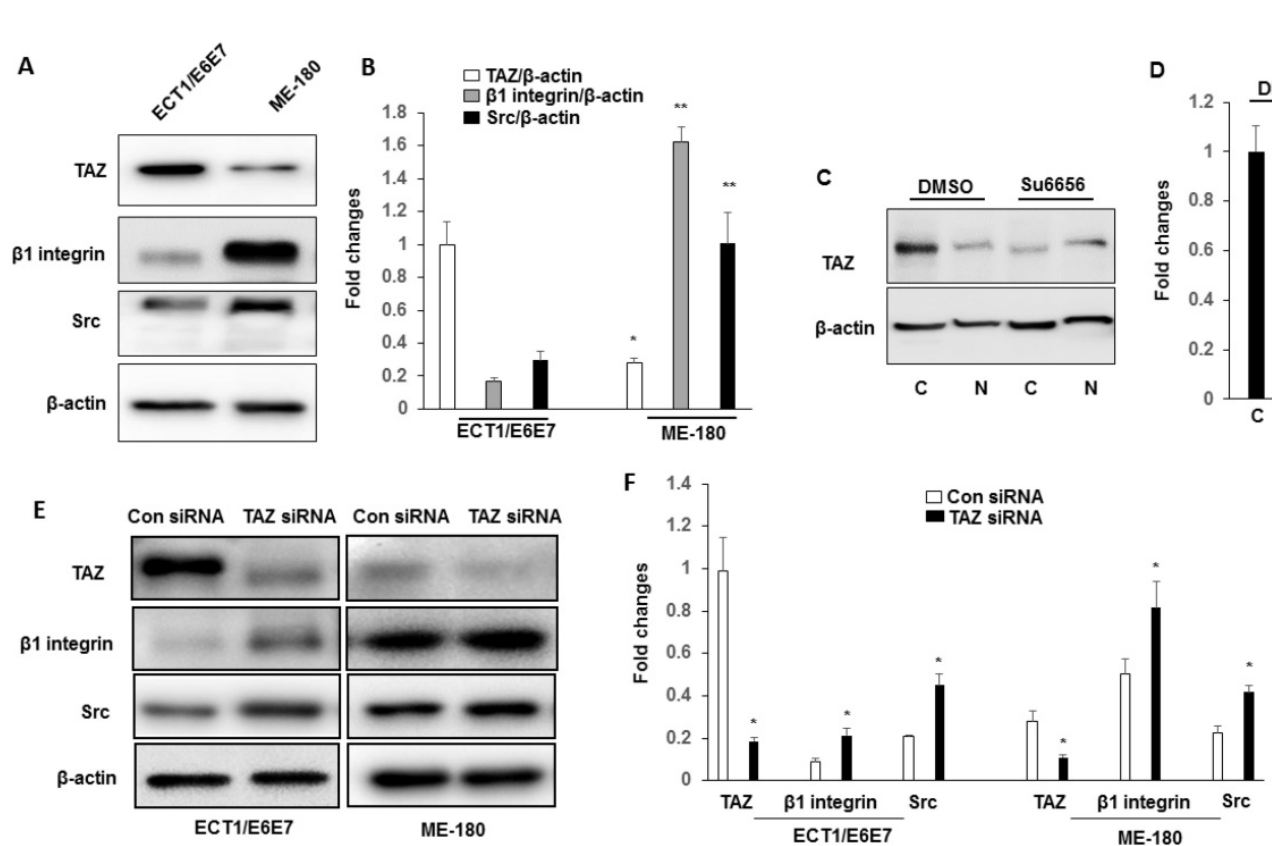

Figure 3. Western blot analysis of TAZ, $\beta 1$ integrin and Src expression in ECT1/E6E7 and ME-180 cell lines. A-B. Cell lysates extracted from these cell lines were subjected to western blot using anti-TAZ, anti- $\beta 1$ integrin and anti-Src antibodies. $* P<0.05$ vs TAZ from ECT1/E6E7; **P<0.05 vs $\beta 1$ integrin and Src from ECT1/E6E7. C-D. Cytosolic and Nuclear TAZ in ME180 cell line with su6656 (1 MM). Nuclear and cytosolic fractions were subjected to western blot analysis to detect TAZ. The levels of $\beta$-actin were used as internal protein loading controls. C: cytosolic; N: nucleus. $* P<0.05$ vs cytosolic by DMSO; $* * P<0.05$ vs nucleus by DMSO. E-F. Both ECT1/E6E7 and ME-180 cells were transfected with a TAZ siRNA or an Src scramble control for $48 \mathrm{~h}$, and changes in the expression of TAZ, $\beta 1$ integrin, and Src were determined by Western blotting relative to the level of $\beta$-actin. $* P<0.05$ vs relative control siRNA. All graphs correspond to the blots above them and represent densitometric analyses of 3 independent experiments. 

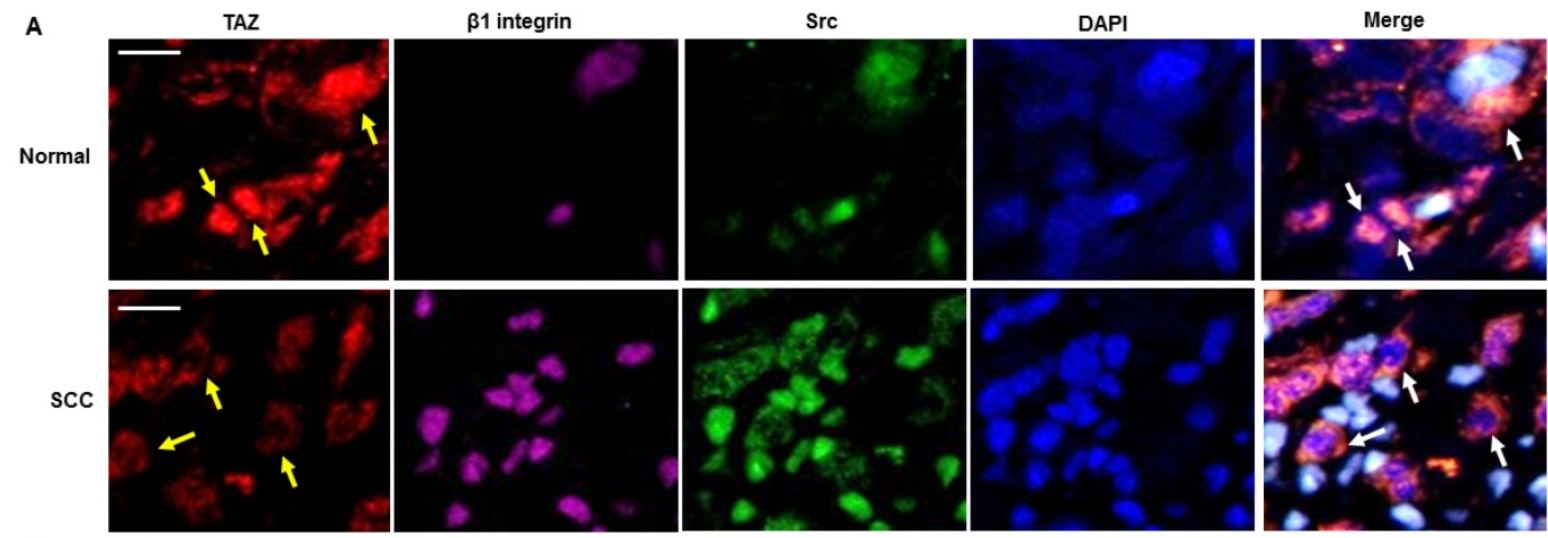

B
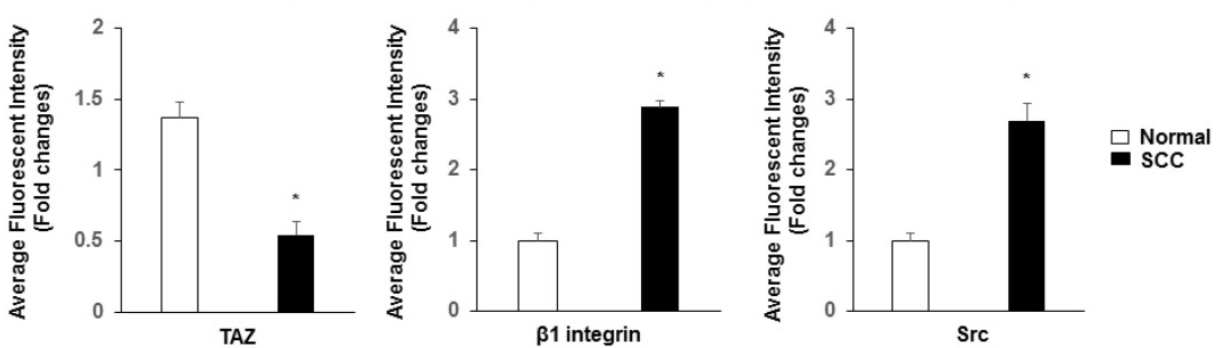

Figure 4. Subcellular localization of TAZ, $\beta 1$ integrin and Src. A. Confocal analysis of nuclear accumulation of TAZ, $\beta 1$ integrin and Src in normal cervix and SCC tissues, respectively. Subcellular localization of TAZ (yellow arrow), $\beta 1$ integrin and Src are shown in the merged image. Blue, nuclei stained with DAPI. Yellow spots indicate the interactions of TAZ, $\beta 1$ integrin and Src (white arrow). Scale bar: $20 \mu \mathrm{m}$. B. Quantification of immunofluorescence fluorescent intensity of normal and SCC.

It was demonstrated that the major regulatory effect of the Hippo pathway on TAZ is via modulating the TAZ translocation between cytoplasm and nucleus [23]. TAZ is a very unstable protein and degraded in cells under high cell densities [24]. Therefore, the localization and stability of TAZ could be associated with carcinogenesis and cancer progression. Elevated TAZ expression levels and nuclear localization have been observed in multiple types of human cancers, including breast cancer [22], lung cancer [10], and often correlate with patient prognosis.

Although most studies suggest that the expression of TAZ is associated with poor prognosis and metastasis, some found that YAP1/TAZ activation can repress metastasis [25,26]. After analysing human cervical cancer, Buglioni et al. found that TAZ was expressed in tumour cells $(78 \%)$ and showed relatively lower expression in non-neoplastic cells in the tumour microenvironment, including endothelial cells, non-lymphocytic stromal cells and tumour-infiltrating lymphocytes [11]. However, in our study, TAZ expression was not observed in non-neoplastic cells in the tumour microenvironment and was only detected in tumour cells. In our study, we found that TAZ was expressed in $84.54 \%$ of SCC samples, most of which were located in the cytoplasms of tumour cells. However, we have not tested whether the expression levels of TAZ correlated with their activity. Additionally, we did not observe a statistically significant correlation between TAZ expression/localization and overall survival.

de Cristofaro et al. reported that the immunofluorescence staining observed in the cytoplasm suggests that the dislocation of TAZ might be one of the causes of the malignant phenotype in papillary thyroid carcinoma [24].The cytoplasmic localization of TAZ might be involved in the initiation and/or the progression of neoplasia. Our study demonstrates for the first time that nuclear and cytosolic TAZ localization are correlated with SCC. Because TAZ is a transcriptional coactivator that shuttles between the cytosol and nucleus, we analysed cytosolic and nucleic expression of TAZ between normal and SCC cervices. TAZ was found to translocate into the cytosol in SCC. Although we have not tested whether TAZ expression levels correlate with degradation in this situation, it was recently demonstrated that the TAZ signalling may also be regulated through TAZ degradation [24].

The expression of $\beta 1$ integrin in SCC has been proposed as a link to the clinical stage and the degree of malignancy [13]. We examined the model to study if integrin-Src signalling regulates the TAZ nuclear localization in cultured normal and cervical cancer human cell lines, and found that these signalling molecules are highly expressed in cancer cell line, The inhibition of Src leads to nuclear accumulation of 
TAZ, and Src plays a key role in regulation of the Hippo pathway transcriptional coactivator TAZ. We confirm that the decreased TAZ leads to the activation of $\beta 1$ integrin and Src signalling. Further work is necessary to understand if Src acts primarily by directly phosphorylating TAZ and regulating its downstream signalling. It is currently unknown whether $\beta 1$ integrin expression regulates TAZ signalling and degradation. One possibility might be that the loss of $\beta 1$ integrin inhibits TAZ phosphorylation and subsequent ubiquitin-mediated degradation, leading to increased levels of nuclear TAZ.

Taken together, our data support a model in which $\beta 1$ integrin signals through Src kinases to support the functions of the Hippo pathway. Upon activation, Src disrupts this pathway, resulting in decreased nuclear TAZ accumulation and driving invasive growth (Fig. 5). In conclusion, our data suggest that the expression and localization of TAZ is inversely associated with the progression and metastasis of cervical cancer. Furthermore, we provide evidence that the localization of the TAZ transcription factor is associated with the activation of integrin-Src signalling pathway.

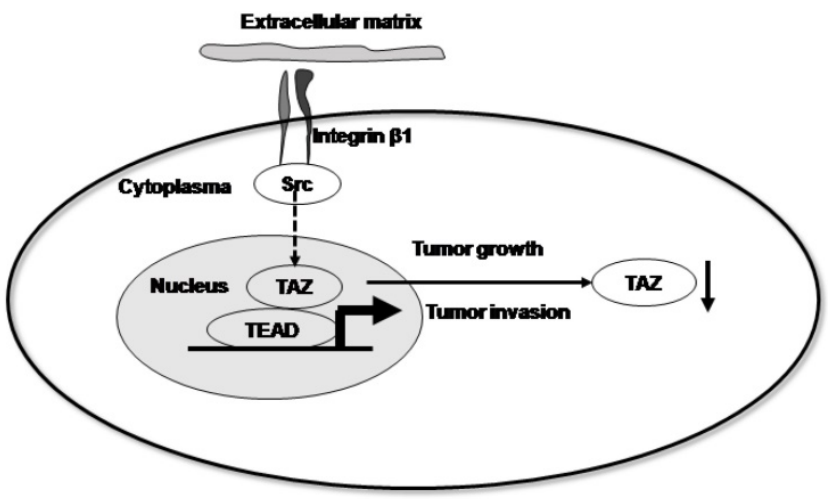

Figure 5. Proposed model by which $\beta 1$ integrin signals through Src kinases to support Hippo pathway functions. Upon activation, Src disrupts this pathway, resulting in decreased nuclear TAZ accumulation and driving invasive growth.

\section{Abbreviations}

CC: cervical cancer; SCC: squamous cell carcinoma; TAZ: transcriptional co-activator with PDZ-binding motif; YAP: Yes-associated protein.

\section{Acknowledgments}

This study was supported by the National Natural Science Foundation of China (grant no. 81172492 to $\mathrm{Hu} \mathrm{L}$ ).

\section{Ethics Committee Approval and Patient Consent}

The study protocol was approved by the
Affiliated Hospital of Southwest Medical University Review Board, and written informed consent was obtained from all study participants. The study was performed in accordance with the principles of Declaration of Helsinki.

\section{Competing Interests}

The authors have declared that no competing interest exists.

\section{References}

1. Jemal A, Bray F, Center MM, et al. Global cancer statistics. CA Cancer J Clin. 2011; 61(2): 69-90.

2. Schiffman M, Castle PE, Jeronimo J, et al. Human papillomavirus and cervical cancer. Lancet 2007; 370:890-07.

3. Doeberitz Mv, Vinokurova S. Host factors in HPV-related carcinogenesis: cellular mechanisms controlling HPV infections. Arch Med Res. 2009;40(6): 435-42.

4. Pan D. The hippo signaling pathway in development and cancer. Dev Cell. 2010;19(4): 491-05.

5. Dong J, Feldmann G, Huang J, et al. Elucidation of a universal size-control mechanism in Drosophila and mammals. Cell 2007;130(6): 1120-33.

6. Zeng $\mathrm{Q}$, Hong $\mathrm{W}$. The emerging role of the hippo pathway in cell contact inhibition, organ size control, and cancer development in mammals. Cancer Cell 2008;13(3):188-92.

7. Zender L, Spector MS, Xue W, et al. Identification and validation of oncogenes in liver cancer using an integrative oncogenomic approach. Cell 2006;125(7): 1253-67.

8. Chan SW, Lim CJ, Guo K, et al. A role for TAZ in migration, invasion, and tumorigenesis of breast cancer cells. Cancer Res. 2008;68(8): 2592-98.

9. Bhat KP, Salazar KL, Balasubramaniyan $\mathrm{V}$, et al. The transcriptional coactivator TAZ regulates mesenchymal differentiation in malignant glioma. Genes Dev. 2011;25(24): 594-09.

10. Xie M, Zhang L, He CS, et al. Prognostic significance of TAZ expression in resected non-small cell lung cancer. J Thorac Oncol. 2012;7(5): 799-07.

11. Buglioni S, Vici P, Sergi D, et al. Analysis of the hippo transducers TAZ and YAP in cervical cancer and its microenvironment. Oncoimmunology. 2016;5(6):e1160187.

12. Brakebusch C, Bouvard D, Stanchi F, et al. Integrins in invasive growth. J Clin Invest. 2002;109(8): 999-1006.

13. Zhan P, Liu L, Liu B, et al. Expression of integrin $\beta 1$ and its significance in squamous cell carcinoma of the cervix. Mol Med Rep. 2014;9(6): 2473-78.

14. Tang Y, Rowe RG, Botvinick EL, et al. MT1-MMP-dependent control of skeletal stem cell commitment via a 1-integrin/YAP/TAZ signaling axis. Dev Cell. 2013;25(4): 402-16.

15. Elbediwy A, Vincent-Mistiaen ZI, Spencer-Dene B, et al. Integrin signaling regulates YAP and TAZ to control skin homeostasis. Development 2016; 143(10): 1674-87.

16. Bouvier C, Macagno N, Nguyen $Q$, et al. Prognostic value of the hippo pathway transcriptional coactivators YAP/TAZ and $\beta 1$-integrin inconventional osteosarcoma. Oncotarget 2016;7(40): 64702-64710.

17. Sykes JA, Whitescarver J, Jernstrom P, et al. Some properties of a new epithelial cell line of human origin. J Natl Cancer Inst. 1970;45(1): 107-22.

18. Fichorova RN, Rheinwald JG, Anderson DJ. Generation of papillomavirus-immortalized cell lines from normal human ectocervical, endocervical, and vaginal epithelium that maintain expression of tissue specific differentiation proteins. Biol Reprod. 1997;57(4): 847-55.

19. Wu J, Strawn TL, Luo $\mathrm{M}$, et al. Plasminogen activator inhibitor-1 inhibits angiogenic signaling by uncoupling vascular endothelial growth factor receptor-2-aV $\beta 3$ integrin cross talk. Arterioscler Thromb Vasc Biol. 2015;35(1):111-20.

20. He C, Mao D, Hua G, et al. The Hippo/YAP pathway interacts with EGFR signaling and HPV oncoproteins to regulate cervical cancer progression. EMBO Mol Med. 2015;7(11):1426-49.

21. Bartucci M, Dattilo R, Moriconi C, et al. TAZ is required for metastatic activity and chemoresistance of breast cancer stem cells. Oncogene 2015;34(6): 681-90.

22. Díaz-Martín J, López-García MÁ, Romero-Pérez L, et al. Nuclear TAZ expression associates with the triple-negative phenotype in breast cancer. Endocr Relat Cancer. 2015; 22(3): 443-54.

23. Lei QY, Zhang H, Zhao B, et al. TAZ promotes cell proliferation and epithelial-mesenchymal transition and is inhibited by the hippo pathway. Mol Cell Biol. 2008;28(7): 2426-36.

24. Liu CY, Zha ZY, Zhou X, et al. The hippo tumor pathway promotes TAZ degradation by phosphorylating a phosphodegron and recruiting the SCF \{beta\}-TrCP E3 ligase. J Biol Chem. 2010; 285(48): 37159-37169.

25. de Cristofaro T, Di Palma T, Ferraro A, et al. TAZ/WWTR1 is overexpressed in papillary thyroid carcinoma. Eur J Cancer. 2011;47(6): 926-33. 
26. Yu SJ, Hu JY, Kuang XY, et al. MicroRNA-200a promotes anoikis resistance and metastasis by targeting YAP1 in human breast cancer. Clin Cancer Res. 2013;19:1389-99.

27. Sun Z, Schwenzer A, Rupp T, et al. Tenascin-C promotes tumor cell migration and metastasis through integrin a9 1 1-mediated YAP inhibition. Cancer Res. 2018;78:950-61. 